\title{
AN ASCOLI THEOREM FOR MULTI-VALUED FUNCTIONS
}

\author{
VINCENT J. MANCUSO \\ (Received 17 March 1969; revised 3 February 1970) \\ Communicated by E. Strzelecki
}

\section{Introduction}

The concept of simultaneous or collective continuity of a family of single valued functions was introduced by Gale [3] for regular spaces to replace equicontinuicy in metric spaces. Smithson [6] extended the standard point-open and compact-open function space topologies to include multi-valued functions. The aim of this paper is to use these topologies and extend the notion of collective continuity in order to obtain an Ascoli type theorem for multi-valued functions analogous to Theorem 1 in [3, p. 304]. We have the following theorem in mind:

THEOREM. Let $X$ be a $T_{2} k$-space and $Y$ a normal $T_{2}$ space. Let $\mathscr{C}=\left\{f \in Y^{m X}\right.$; $f$ is continuous and point-compact $\}$ have the compact-open topology. Then a pointlike family $\mathscr{F} \subset \mathscr{C}$ is compact if and only if

(a) $\mathscr{F}$ is closed in $\mathscr{C}$

(b) $\mathscr{F}[x]$ is compact for each $x \in X$, and

(c) Every closed subset of $\mathscr{F}$ is collectively continuous

(See Theorem 4.5).

\section{Preliminaries}

Let $X$ and $Y$ be non-empty sets. If $f(x)$ is a non-empty subset of $Y$ for each $x \in X$, we say $f$ is a multi-valued function or multifunction from $X$ to $Y$ and write $f: X \rightarrow Y$. If $Y$ carries a topology and $f(x)$ is a non-empty closed (compact) subset of $Y$ for each $x \in X$, we say that $f$ is point-closed (point-compact). We will write $f x$ instead of $f(x)$, and $\bar{A}=$ the closure of $A$.

Remark 2.1. Let $P(Y)$ be the set of all non-empty subsets of $Y$. Clearly a multi-valued function $f: X \rightarrow Y$ induces a single-valued $f^{\prime} \in(P(Y))^{X}$ by $f^{\prime}(x)=f x$. Now let $R \subset X \times Y$ be a relation, $A \subset X$ and

$$
A R=\{y \in Y ;(x, y) \in R \text { for some } x \in A\} .
$$

Since there is a natural bijection between $(P(Y))^{X}$ and the set of all relations 
$R \subset X \times Y$ such that $Y R^{-1}=X$, one may think of a multi-valued function, more fundamentally perhaps, as such a relation. The reader is referred to [2] for further discussion along these lines.

Let $f: X \rightarrow Y$ be a multifunction. If $A \subset X, f(A)=\cup\{f x ; x \in A\}$. If $B \subset Y$, $f^{-} B=\{x ; f x \cap B \neq \emptyset\}$ and $f^{+} B=\{x ; f x \subset B\}$. We will adopt the notion of continuity of a multifunction as given in [7]:

Definition 2.2. Let $X$ and $Y$ be spaces. Then $f: X \rightarrow Y$ is continuous on $X$ if for each $x \in X$, (1) and (2) hold:

(1) Given $U$ open in $Y$ such that $f x \cap U \neq \emptyset$, there exists a neighborhood $V$ of $x$ in $X$ such that $f z \cap U \neq \emptyset$ for all $z \in V$ (Equivalently, $f^{-} U$ is open in $X$ when $U$ is open in $Y$ ).

(2) Given $U$ open in $Y$ such that $f x \subset U$, there exists a neighborhood $V$ of $x$ in $X$ such that $f(V) \subset U$. (Equivalently, $f^{+} U$ is open in $X$ when $U$ is open in $Y$ ).

REMARK 2.3. If $f: X \rightarrow Y$ is point-closed and $S(Y)$ is the set of non-empty closed subsets of $Y$, we can give $S(Y)$ the Vietoris topology in which a basic open set is of the form

$$
\left\{A \in S(Y) ; A \subset \cup U_{i} \text { and } A \cap U_{i} \neq \emptyset, i=1, \cdots, n\right\}
$$

where $U_{i}$ is open in $Y$ for each $i$. Now $f$ induces a single-valued $\operatorname{map} f^{*}(S(Y))^{X}$ by $f^{*}(x)=f x$. We can then define $f$ to be continuous provided $f^{*}$ is continuous. This is also equivalent to Definition 2.2 (See [7] and [8]). In [5, p. 120], one finds that Definition 2.2 is the same as strong continuity and that Ponomarev defines $f$ to be continuous provided that $f$ satisfies only (2) of Definition 2.2. Berge [1, p. 109] and Day and Franklin [2, p. 289] additionally require $f$ to be point-compact.

The following lemmas, though elementary, will be crucial in later results.

LEMMA 2.4. Let $f: X \rightarrow Y$ be a point-closed continuous multifunction from $X$ to a normal $T_{2}$ space $Y$. If $f x \cap U \neq \emptyset$ for some $U$ open in $Y$, there exists a neighborhood $V$ of $x$ in $X$ such that $f z \cap U \neq \emptyset$ for all $z \in \bar{V}$.

Proof. Let $y \in f x \cap U$. By regularity, choose $W$ open in $Y$ such that $y \in W$ and $\bar{W} \subset U$. Since $f$ is continuous, $f v \cap W \neq \emptyset$ for all $v$ in some neighborhood $V$ of $x$ in $X$. Let $z \in \bar{V}$ and suppose $f z \cap U=\emptyset$. Then $f z \cap \bar{W}=\emptyset$ and so by normality, we can find $G$ open in $Y$ such that $f z \subset G$ and $\bar{G} \cap \bar{W}=\emptyset$. Again by continuity, there exists a neighborhood $H$ of $z$ in $X$ such that $f(H) \subset G$. Since $z \in \bar{V}$, pick a $v \in V \cap H$. Then $f v \cap W \neq \emptyset$ and $f v \subset G$, a contradiction, since $\bar{G} \cap \bar{W}=\emptyset$.

A multifunction satisfying Definition $2.2(1)$ is called lower semi-continuous. The following lemma appears as Lemma 1 in [6]:

Lemma 2.5. Let $f: X \rightarrow Y$ be lower semi-continuous from $X$ to a regular space 
$Y$. If $K \subset X$ is compact, and if $f x \cap V \neq \emptyset$ for all $x \in K$, where $V$ is an open subset of $Y$, then there exists an open set $W$ in $Y$ such that $\bar{W} \subset V$ and $f x \cap W \neq \emptyset$ for all $x \in K$.

\section{Point-open and compact-open topologies}

In [6], the concept of a product space is extended to include multi-valued functions: Let $X$ be a non-empty set and $\left\{Y_{x} ; x \in X\right\}$ a family of sets indexed by $X$. A multifunction

$$
f: X \rightarrow \cup\left\{Y_{x} ; x \in X\right\}
$$

is called an $m$-choice function on $X$ if $f x \subset Y_{x}$ for each $x \in X$. The $m$-product,

$$
P\left\{Y_{x} ; x \in X\right\}=\{f ; f \text { is an } m \text {-choice function on } X\} \text {. }
$$

The projections $p_{x}$ defined by $p_{x}(f)=f x$ are now multifunctions from the $m$ product to $Y_{x}$. In case $Y_{x}=Y$ for each $x \in X$, the $m$-product is simply written $Y^{m X}$.

As in the case of single-valued functions [4, p. 90], if each $Y_{x}$ is given a topology, the $m$-product topology will be the smallest topology on the $m$-product such that all the projections are continuous. Therefore, with Definition 2.2 in mind, the following definitions become natural.

Definition 3.1. Let $U$ and $V$ be open in $Y$. The m-product topology $\mathscr{P}^{\prime}$ on $Y^{m X}$ is that topology having all sets of the form

as a subbasis.

$$
\{f ; f x \subset U\} \text { or }\{f ; f x \cap V \neq \emptyset\}
$$

DEFINITION 3.2. Let $\mathscr{F} \subset Y^{m X}$. The topology of pointwise convergence or the point-open topology $\mathscr{P}$ on $\mathscr{F}$ is $\mathscr{P}$ ' relativized to $\mathscr{F}$.

Again, as with the single-valued case, we can broaden the concept of nearness of functions by requiring that if $f$ behaves a certain way on compact sets, then functions near $f$ must also behave in this way. The smallest topology compatible with this requirement is the compact-open topology [4, p. 221]. We therefore make the following definition for multi-valued functions.

Definition 3.3. Let $\mathscr{F} \subset Y^{m X}, A \subset X$ compact and $U, V$ open in $Y$. The compact-open topology $\mathscr{K}$ is that topology on $\mathscr{F}$ having all sets of the form

$$
\{f \in \mathscr{F} ; f(A) \subset U\} \text { or }\{f \in \mathscr{F} ; f x \cap V \neq \emptyset \text { for all } x \in A\}
$$

as a subbasis.

REMARK 3.4. The reader is referred to [6] for further discussion and motivation of the topologies $\mathscr{P}$ and $\mathscr{K}$. Reference [2, Prop. 1] is suggested for the treatment of the compact-open topology in the language of relations. 


\section{Collective continuity}

Since a multifunction admits two types of inverse images, we must modify Gale's definition of collective or simultaneous continuity so that we may include multifunctions (see the parenthetical remark after Theorem 1, p. 304 in [3]).

Definition 4.1. Let $\mathscr{F} \subset Y^{m X}$ and $B \subset Y$.

(1) $\bigcup \mathscr{F}^{-}(B)=\bigcup\left\{f^{-} B ; f \in \mathscr{F}\right\}$ and $\bigcup \mathscr{F}^{+}(B)=\bigcup\left\{f^{+} B ; f \in \mathscr{F}\right\}$.

(2) $\bigcap_{\mathscr{F}^{-}}(B)=\bigcap\left\{f^{-} B ; f \in \mathscr{F}\right\}$ and $\bigcap_{\mathscr{F}^{+}}(B)=\bigcap\left\{f^{+} B ; f \in \mathscr{F}\right\}$.

DefinITION 4.2. A family $\mathscr{F} \subset Y^{m X}$ is collectively continuous provided $\bigcup \mathscr{F}^{-}(B)$ and $\bigcup \mathscr{F}^{+}(B)$ are closed in $X$ whenever $B$ is closed in $Y$, or equivalently $\bigcap \mathscr{F}^{-}(B)$ and $\bigcap \mathscr{F}^{+}(B)$ are open in $X$ whenever $B$ is open in $Y$.

Lemma 4.3. Let $\mathscr{C}=\left\{f \in Y^{m x} ; f\right.$ is continuous and point-compact $\}$ have the $\mathscr{K}$-topology, and let $Y$ be a regular Hausdorff space. If $\mathscr{F} \subset \mathscr{C}$ is such that each closed subset of $\mathscr{F}$ is collectively continuous, then $\mathscr{P}=\mathscr{K}$ on $\mathscr{F}$.

Proof. Clearly $\mathscr{P} \subset \mathscr{K}$ by Definitions 3.2 and 3.3.

Let

$$
G=\{f \in \mathscr{C} ; f(A) \subset U \text { and } f x \cap V \neq \emptyset \text { for } x \in A\},
$$

where $A$ is compact in $X$ and $U, V$ are open in $Y$. We show that $G$ is open in $(\mathscr{F}, \mathscr{P})$ :

Let $f \in G \cap \mathscr{F}$. Then $f(A)$ is compact $[1$, p. 110]. Since $Y$ is regular, there exists $H$ open in $Y$ such that $f(A) \subset H$ and $\bar{H} \subset U$. For each $x \in A, f x \cap V \neq \emptyset$ and so by Lemma 2.5 , there exists $V^{\prime}$ open in $Y$ such that $\overline{V^{\prime}} \subset V$ and $f x \cap V^{\prime} \neq \emptyset$ for all $x \in A$.

For each $x \in A$, define $W(x)=\left\{g \in \mathscr{F} ; g x \subset H\right.$ and $\left.g x \cap \overline{V^{\prime}} \neq \emptyset\right\}$. Then $W(x)$ is closed in $(\mathscr{F}, \mathscr{P})$ and hence in $(\mathscr{F}, \mathscr{K})$, and non-empty since $f \in W(x)$. By collective continuity, the sets

$$
N(x)=\cap W(x)^{-}(V) \text { and } M(x)=\cap W(x)^{+}(U)
$$

are open in $X$ and contain $x$. Let $0(x)=N(x) \cap M(x)$ for each $x \in A$. Since $A$ is compact, there exist $x_{1}, \cdots, x_{n} \in A$ such that $A \subset \bigcup\left\{0\left(x_{i}\right) ; i=1, \cdots, n\right\}$.

For each $i$, let $G_{i}=\left\{g \in \mathscr{F} ; g x_{i} \subset H\right.$ and $\left.g x_{i} \cap V^{\prime} \neq \emptyset\right\}$. Clearly

$$
f \in \bigcap\left\{G_{i} ; i=1, \cdots, n\right\},
$$

and so it suffices to show that $\bigcap\left\{G_{i} ; i=1, \cdots, n\right\} \subset G$. Suppose $g x_{i} \subset H$ and $g x_{i} \cap V^{\prime} \neq \emptyset$ for each $i$. If $x \in A, x \in 0\left(x_{i}\right)$ for some $i$, and so $g x \subset U$ and $g x \cap V^{\prime} \neq \emptyset$, i.e., $g \in G$ and the proof is complete.

NotATiOn. Let $\mathscr{F} \subset Y^{m X}$ and $x \in X . \mathscr{F}[x]=\bigcup\{f x ; f \in \mathscr{F}\}$.

Definition 4.4. Let $\mathscr{F} \subset Y^{m X}$ be a point-closed family and let $\overline{\mathscr{F}}$ ba the 
$\mathscr{P}$-closure of $\mathscr{F}$ in the set of all point-closed multifunctions in $Y^{m X}$. Then $\mathscr{F}$ is called point-like if whenever $g \in \overline{\mathscr{F}}$ and $g x_{0} \subset \mathscr{F}\left[x_{0}\right]$, there exists an $f \in \mathscr{F}$ such that $f x_{0}=g x_{0}$.

THEOREM 4.5. Let $X$ be a $T_{2} k$-space and $Y$ a normal $T_{2}$ space. Let $\mathscr{C}=\left\{f \in Y^{m X}\right.$; $f$ is continuous and point-compact $\}$ have the $\mathscr{K}$-topology. Then a point-like family $\mathscr{F} \subset \mathscr{C}$ is compact if and only if

(a) $\mathscr{F}$ is closed in $\mathscr{C}$

(b) $\mathscr{F}[x]$ is compact for each $x \in X$, and

(c) Every closed subset of $\mathscr{F}$ is collectively continuous.

Proof. Necessity. If $\mathscr{F}$ is compact, the $\mathrm{n}(\mathrm{a})$ follows since $(\mathscr{C}, \mathscr{K})$ is $T_{2}[6$, Prop. 6]. (b) follcws since $\mathscr{F}[x]=p_{x}(\mathscr{F})$ and each $p_{x}$ is continuous. Now since $\mathscr{F}$ is compact, any closed subset of $\mathscr{F}$ will also be compact and so it is sufficient to prove (c) for $\mathscr{F}$ itself, i.e., we show that $\mathscr{F}$ is collectively continous. Let $B$ be closed in $Y$ and $A$ compact in $X$. Since $X$ is a $k$-space [4, p. 230], we need only show that the sets $S=\left(\bigcup \mathscr{F}^{-}(B)\right) \cap A$ and $S^{\prime}=\left(\bigcup \mathscr{F}^{+}(B)\right) \cap A$ are relatively closed. We handle $S^{\prime}$ first: Suppose $z \notin S^{\prime}$. Note that the non-trivial case will occur when $z \in A$. It follows that $z \notin \bigcup \mathscr{F}^{+}(B)$, and so $f z \cap(Y-B) \neq \emptyset$ for each $f \in \mathscr{F}$. By Lemma 2.4, there $\epsilon$ xists a neighborhood $N(f)$ of $z$ in $X$ such that $f z \cap$ $(Y-B) \neq \emptyset$ for all $x \in \overline{N(f)}$.

Now $B(f)=\overline{N(f)} \cap A$ is compact and so the set

$$
W(f)=\{g ; g x \cap(Y-B) \neq \emptyset \text { for all } x \in B(f)\}
$$

is open in $(\mathscr{F}, \mathscr{K})$. Since $f \in W(f)$, the collection $\{W(f) ; f \in \mathscr{F}\}$ covers $\mathscr{F}$. Since $\mathscr{F}$ is compact, there exist $f_{1}, \cdots, f_{n} \in \mathscr{F}$ such that $\left\{W\left(f_{i}\right) ; i=1, \cdots, n\right\}$ covers $\mathscr{F}$.

Let $B^{*}=\cap\left\{B\left(f_{i}\right) ; i=1, \cdots, n\right\}$. Then for each $f \in \mathscr{F}, f x \cap(Y-B) \neq \emptyset$ for all $x \in B^{*}$ and so $B^{*} \cap\left(\bigcup \mathscr{F}^{+}(B)\right)=\emptyset$. If we let

$$
N^{*}=\cap\left\{N\left(f_{i}\right) ; i=1, \cdots, n\right\},
$$

then $N^{*} \cap A$ is a neighborhood of $z$ in $A$ which does not meet $S^{\prime}$ (note that $N^{*} \cap A \subset B^{*}$ ). Therefore $z$ is not in the relative closure of $S^{\prime}$, and so $S^{\prime}$ is relatively closed.

To show that $S$ is relatively closed, we must modify the above argument. Suppose $z \notin S$. Again, we need only consider the case when $z \in A$. Then $z \notin \bigcup \mathscr{F}^{-}(B)$, and so $f z \subset Y-B$ for each $f \in \mathscr{F}$. Since each $f$ is continuous, we may use the normality of $Y$ to find a neighbcrhood $N(f)$ of $z$ in $X$ such that $\overline{f(N(f))} \subset Y-B$. As before, $B(f)=\overline{N(f)} \cap A$ is compact, and so the set

$$
W(f)=\{g \in \mathscr{F} ; g(B(f)) \subset Y-B\}
$$

is cpen in $(\mathscr{F}, \mathscr{K})$. Now since $f(\overline{N(f)}) \subset \overline{f(N(f))}[5$, p. 120], the collection $\{W(f) ; f \in \mathscr{F}\}$ covers $\mathscr{F}$. The rest of the argument is similar to that given above. 
Sufficiency. Since the family $\mathscr{F}$ satisfies (c), by Lemma 4.3 we may consider $\mathscr{F}$ embedded in $(\mathscr{C}, \mathscr{P})$. By (b), the $m$-product $P\{\mathscr{F}[x] ; x \in X\}$ is $\mathscr{P}$-compact, and since $\mathscr{F} \subset P\{\mathscr{F}[x] ; x \in X\}$, it suffices to prove that $\mathscr{F}$ is closed in $(\mathscr{C}, \mathscr{P})$. Arguing as in $[3$, p. 306], this will be accomplished by showing that $\mathscr{F} \subset \mathscr{C}$, where $\overline{\mathscr{F}}$ denotes the $\mathscr{P}$-closure of $\mathscr{F}$ in the sel of point-closed members of $Y^{\boldsymbol{m} x}$.

Let $g \in \overline{\mathscr{F}}$. We show $g$ is continuous and point-compact: Since $\overline{\mathscr{F}}$ is contained in the $m$-product $P\{\mathscr{F}[x] ; x \in X\}$, and $\mathscr{F}$ is point-like, $g$ is point-compact (note that every $f \in \mathscr{F}$ is point-compact). We now verify continuity.

Let $z \in X$ and $g z \subset U$ where $U$ is open in $Y$. By repeated application of normality, we can find $G$ and $G^{\prime}$ open in $Y$ such that $g z \subset G, \bar{G} \subset G^{\prime}$ and $\overline{G^{\prime}} \subset U$. Let $T=\{f \in \mathscr{F} ; f z \subset \bar{G}\}$. Then $T$ is $\mathscr{P}$-closed and non-empty since $\mathscr{F}$ is point-like. By collective continuity, $N(z)=\bigcap T^{+}\left(G^{\prime}\right)$ is a neighborhood of $z$ in $X$. We claim $g(N(z)) \subset U$. Let $x \in N(z)$ and let $W$ be any neighborhood of $y \in g x$. The set

$$
M=\{f ; f z \subset G\} \cap\{f ; f x \cap W \neq \emptyset\}
$$

is a $\mathscr{P}$-neighborhood of $g$ and so there exists an $f^{\prime} \in M \cap \mathscr{F}$. Since $f^{\prime} \in T$, $f^{\prime} x \subset G^{\prime}$ and $f^{\prime} x \cap W \neq \emptyset$. Therefore $W \cap G^{\prime} \neq \emptyset$, and since $y$ was chosen arbitrarily in $g x$, we have $g x \subset \overline{G^{\prime}} \subset U$.

Suppose $g z \cap V \neq \emptyset$ where $V$ is open in $Y$. Let $y \in g z \cap V$. By regularity, find $G$ cpen in $Y$ such that $y \in G$ and $\bar{G} \subset V$. By normality, find $G^{\prime}$ open in $Y$ such that $\bar{G} \subset G^{\prime}$ and $\overline{G^{\prime}} \subset V$. As before, $T=\{f \in \mathscr{F} ; f z \cap \bar{G} \neq \emptyset\}$ is non-empty since $\mathscr{F}$ is point-like, and since $T$ is $\mathscr{P}$-closed, $M(z)=\bigcap T^{-}\left(G^{\prime}\right)$ is a neighborhood of $z$ by collective continuity. We claim that $g x \cap V \neq \emptyset$ for all $x \in M(z)$. Suppose $g x \cap V=\emptyset$ for some $x \in M(z)$. Then $g x \cap \overline{G^{\prime}}=\emptyset$, and so by normality there exists $H$ open in $Y$ such that $g x \subset H$ and $\bar{H} \cap \overline{G^{\prime}}=\emptyset$. Now since the set

$$
M=\{f ; f x \subset H\} \cap\{f ; f z \cap G \neq \emptyset\}
$$

is a $\mathscr{P}$-neighborhood of $g$, there $\epsilon$ xists an $f^{\prime} \in M \cap \mathscr{F}$. Then $f^{\prime} \in T$ and so we bave $f^{\prime} x \cap G^{\prime} \neq \emptyset$, a contradiction, since $f^{\prime} x \subset H$ and $\bar{H} \cap \overline{G^{\prime}}=\emptyset$. We have shown that $g$ satisfies Definition 2.2 and the proof is complete.

COROLLARY 4.6. Let $\mathscr{F}$ be a point-compact family of continuous multifunctions from a $T_{2} k$-space to a normal $T_{2}$ space $Y$, and let $\mathscr{F}$ have the $\mathscr{K}$-topology. Then the map $\Phi: X \rightarrow\left(Y^{m \mathscr{F}}, \mathscr{K}\right)$ defined by $\Phi(x) f=f x$ is continuous.

Proof. Let

$$
G=\{\sigma ; \sigma(A) \subset U \text { and } \sigma(f) \cap V \neq \emptyset \text { for each } f \in A\}
$$

where $A$ is compact in $(\mathscr{F}, \mathscr{K})$ and $U, V$ are open in $Y$. Suppose $\Phi(x) \in G$. Let $N(x)=\bigcap A^{+}(U)$ and $M(x)=\bigcap A^{-}(V)$. By Theorem 4.5, $N(x) \cap M(x)$ is a neighborhood of $x$. If $z \in N(x) \cap M(x), f z \subset U$ and $f z \cap V \neq \emptyset$ for each $f \in A$. Hence $\Phi(z) \in G$ and so $\Phi(N(x) \cap M(x)) \subset G$. 
REMARK 4.7. Although the proofs in this section parallel those given in [3] for the single valued case, some nontrivial modifications had to be made. For example, the reader is directed to the crucial roles played by Lemmas 2.4 and 2.5 in our main results. Note that the hypothesis that $\mathscr{F}$ be point-like in Theorem 4.5 doe not lose the single-valued functions. Moreover, it does not seem likely that it can be dropped since it not only guaranteed that the sets $T$ be nonempty, but it insured that each $g \in \overline{\mathscr{F}}$ be point-compact (and hence point-closed) so that the full force of normality of $Y$ could be used. Finally, although Theorem 4.5 can't be considered as a true extension of Gale's theorem since we require $Y$ to be normal $T_{2}$ and not just regular, it is frequently necessary to assume the next stronger separation axiom in order to generalize many results from single-valued to multi-valued functions.

\section{References}

[1] C. Berge, Topological Spaces (New York, 1963).

[2] J. M. Day and S. P. Franklin, 'Spaces of continuous relations', Math. Annalen 167 (1967), 289-292

[3] D. Gale, 'Compact sets of functions and function rings', Proc. Amer. Math. Soc. 43 (1950), 303-308.

[4] J. L. Kelley, General Topology (Princeton, 1955).

[5] V. I. Ponomarev, 'Properties of topological spaces preserved under multi-valued continuous mappings', Amer. Math. Soc. Transl. (series 2), 38 (1964), 119-140.

[6] R. E. Smithson, 'Topologies on sets of relations', to appear.

[7] R. E. Smithson, 'Some general properties of multi-valued functions', Pac. J. Math. 15 (1965), 681-703.

[8] W. Strother, 'Fixed points, fixed sets, and M-retracts', Duke J. Math. 22 (1955), 551-556.

St. John's University

Jamaica, New York

U.S.A. 